\title{
Correction to: Evaluation and Validation
}

\section{Correction to:}

Chapter 5 in: P. Marwedel, Embedded System Design, Embedded Systems,

https://doi.org/10.1007/978-3-030-60910-8_5

This chapter was published with the incorrect caption for the second part of Fig. 5.25. This has now been updated (on page no. 277) as follows:

Fig. 5.25 (continued): 100\% utilization 